\title{
Efeito da variabilidade de resíduos madeireiros na produção e qualidade de briquetes
}

\author{
Rosimeire Cavalcante dos Santos ${ }^{1}$ Angélica de Cássia Oliveira Carneiro ${ }^{2}$ Gracielle Raissa Fernandes Damasceno ${ }^{1}$ \\ Ana Flávia Neves Mendes Castro ${ }^{3}$ Renato Vinícius Oliveira Castro ${ }^{3}$ Lidiomar Soares da Costa ${ }^{4}$ Sara Esther de \\ Lima Costa ${ }^{1}$
}

${ }^{1}$ Universidade Federal do Rio Grande do Norte, RN 160, KM 03, Distrito de Jundiaí, Macaíba-RN, 59280-000
${ }^{2}$ Universidade Federal de Viçosa, Avenida Peter Henry Rolfs, s/n - Campus Universitário, Viçosa - MG, 36570-900
${ }^{3}$ Universidade Federal de São João Del Rey, Campus Sete Lagoas, Rodovia MG 424 - Km 47, Sete Lagoas-MG, 35701-970
${ }^{4}$ Universidade Federal de Uberlândia, Campus Monte Carmelo, Rodovia LMG 746, km 1, Monte Carmelo-MG, 38500-000

*Author for correspondence: Lidiomar.ef@gmail.com

Received: August 2018 / Accepted: February 2019 / Published: 31 March 2019

\section{Resumo}

Objetivou-se nesse estudo caracterizar quimicamente e fisicamente resíduos madeireiros utilizados para produzir briquetes, além de relacionar essas características com ao potencial de utilização dos briquetes para geração de energia em cerâmicas do estado do Rio Grande do Norte. Resíduos oriundos de madeiras de diversas serrarias foram coletados de três diferentes pontos de uma pilha de estocagem e, utilizando peneiras, foi classificado nas seguintes granulometrias: $\geq 9,50 \mathrm{~mm} ;<9,5 \mathrm{~mm}$ e $\geq 4,75 \mathrm{~mm} ;<4,75$ $\mathrm{mm}$ e $\geq 2,36 \mathrm{~mm} ;<2,36 \mathrm{~mm}$ e sem classificação, e então foram analisados quanto à composição química, análise química imediata e poder calorífico superior. Foram produzidos briquetes laboratoriais com resíduos de cada granulometria e briquetes industriais coletados de matriz da briquetadeira formaram outro tratamento, estabelecido como padrão comparativo para aqueles produzidos em laboratório. Os briquetes, tanto laboratoriais quanto industriais, produzidos com a utilização dos resíduos de serrarias, apresentam características como, poder calorífico superior, densidade aparente, resistência à compressão plana, teor de lignina e quantidade de carbono fixo, que os qualificam como importante fonte de energia para as indústrias de cerâmica, em razão dos valores considerados adequados para a biomassa energética. É necessária a adoção de medidas de controle da matéria-prima, como granulometria de resíduos e umidade do material, para que os briquetes sejam eficientes em gerar energia calorífica.

Palavras-chave: Briquetagem, Bioenergia, Energia Térmica.

\footnotetext{
Abstract

This study aimed to characterize chemically and physically wood residues used to produce briquettes, besides relating these characteristics to the potential of the use of briquettes for energy generation in ceramics in Rio Grande do Norte state. Residues from wood from several sawmills were collected from three different points of a storage pile and, using sieves, were classified in the following granulometries: $\geq 9.50 \mathrm{~mm} ;<9.5 \mathrm{~mm}$ and $\geq 4.75 \mathrm{~mm} ;<4.75$ $\mathrm{mm}$ and $\geq 2.36 \mathrm{~mm} ;<2.36 \mathrm{~mm}$ and unclassified, and then analyzed for chemical composition, immediate chemical analysis and higher calorific value. Laboratory briquettes were produced with residues of each granulometry and industrial briquettes collected from the briquetting machine composed another treatment, established as a comparative standard for those produced in the laboratory. Both laboratory and industrial briquettes produced by the use of sawmills residue have characteristics such as higher calorific value, apparent density, flat compressive strength, lignin
}

content and quantity of fixed carbon, which qualify them as an important source of energy for the ceramic industries, due to the values considered adequate for energy biomass. It is necessary to adopt raw material control measures, such as grain size and material moisture, so that the briquettes are efficient in generating thermal energy.

Keywords: Briquetting, Bioenergy, thermal energy.

\section{Introdução}

O bioma Caatinga ocorre de forma predominante na região nordeste do Brasil e é um dos mais degradados, encontrando-se em situação preocupante no que diz respeito ao seu grau de desertificação, pelas próprias condições naturais de clima e vegetação em que o mesmo é submetido, adicionado à intensificação deste processo pela supressão desregrada de seus recursos para atender interesses humanos (Pareyn 2010). Parte das causas da devastação do bioma refere-se à exploração mal planejada que decorre, principalmente, da dependência da madeira como principal fonte de energia no setor domiciliar: casas, queijeiras, casa de farinha; e no setor industrial à exemplo das padarias, pizzarias, e especialmente, do setor cerâmico.

No estado do Rio Grande do Norte, extrações desregradas de espécies nativas para fornecimento de lenha às indústrias ceramistas, bem como à extração da argila dos solos para atender às demandas produtivas desse setor, têm intensificado o processo de desertificação na região do Seridó, que possui como principal atividade econômica à produção de cerâmica vermelha (Costa et al. 2009).

De acordo com o Serviço Brasileiro de Apoio às Micro e Pequenas Empresas - SEBRAE (2013) o polo cerâmico do Seridó possui 99 das 186 empresas produtoras de cerâmica do estado; o município de Parelhas concentra o maior número de cerâmicas nessa região, com 33 unidades de produção, se destacando como maior produtor do mesmo.

Nesse sentido, essa atividade contribui para agravar a susceptibilidade da região à desertificação. A Agência de Desenvolvimento Sustentável do Seridó afirmou que na região do Seridó a maioria das espécies da vegetação da Caatinga era explorada, sendo as principais: Angico (Anadenanthera macrocarpa), Aroeira (Schinus terebinthifolius), Catingueira (Poincianela pyramidalis), Faveleira (Cnidoscolus quercifolius), Jurema (Mimosa hostilis), Marmeleiro (Croton sonderianus), Mufumbo (Combretum leprosum), Pereiro (Aspidosperma pyrifolium), e também algumas que foram introduzidas na região, como: Algaroba (Prosopis juliflora), Cajueiro (Anacardium accidentale) e Aveloz (Euphorbia tirucalli) (Silva 2008).

Tendo em vista a dificuldade da disponibilidade de madeira, principalmente à escassez ocasionada pela 
exploração não sustentável da vegetação da Caatinga, como alternativa ao uso da lenha da vegetação nativa, utiliza-se poda de espécies frutíferas, a exemplo do cajueiro e também resíduos madeireiros oriundos de marcenarias, tanto para queima direta ou para fabricação de briquetes.

Briquetes são biocombustíveis densificados elaborados sob pressões e temperaturas controladas (Silva 2007). Geralmente para a produção de briquetes utilizam-se resíduos heterogêneos, que passam por processo de moagem, secagem e compactação, onde se densifica a biomassa, transformando o resíduo em combustível sólido (Quirino 2007). Sem destinação adequada, estes resíduos podem contaminar o ambiente pela deposição em locais inadequados. Assim, a utilização destes para produção de briquetes seria uma alternativa ambientalmente favorável para o uso dos resíduos.

De acordo com Rodrigues (2010) vários fatores influenciam a qualidade dos briquetes, dentre eles a umidade, densidade aparente, poder calorífico superior, resistência à compressão plana, análise química imediata e composição química da matéria prima de origem para a confecção destes combustíveis.

Assim, o objetivo do presente estudo foi caracterizar quimicamente e fisicamente resíduos madeireiros (serragem) utilizados para produzir briquetes, além de relacionar essas características com ao potencial de utilização dos briquetes para geração de energia em cerâmicas do estado do Rio Grande do Norte.

\section{Material e Métodos}

A coleta das amostras de resíduos madeireiros e dos briquetes foi realizada na cerâmica Bela Vista, localizada no município de Parelhas (6037'56.72”S, 36³9'29.21”W) e altitude média de 546 m, na região do Seridó, Estado do Rio Grande do Norte. A empresa produz cerâmica vermelha utilizando no processo de queima, além de lenha, briquetes fabricados no próprio pátio da fábrica. A mesma adquire resíduos madeireiros de diversas serrarias do entorno para viabilizar a fabricação dos briquetes.

No pátio de fabricação coletou-se $1,0 \mathrm{~kg}$ de resíduo madeireiro de três diferentes pontos da pilha de estocagem do resíduo: na parte superior da pilha; na parte intermediária da pilha e na parte inferior da pilha, totalizando $3 \mathrm{~kg}$ de material. Estes resíduos foram oriundos de madeiras de diversas serrarias no estado do Rio Grande do Norte, localizadas a um raio máximo de $130 \mathrm{~km}$ de distância da cerâmica e não houve identificação das espécies madeireiras. Em seguida, o material foi enviado para o Laboratório de Painéis e Energia da Madeira (LAPEM) da Universidade Federal de Viçosa - UFV, localizado no município de Viçosa - estado de Minas Gerais, e o resíduo foi classificado em peneiras de 40 e 60 mesh, sendo o material retido na peneira de 60 mesh utilizado para a determinação da composição química, análise química imediata e poder calorífico superior.

Para utilização desses resíduos na produção de briquetes laboratoriais, os mesmos passaram por classificação granulométrica com o auxílio de um conjunto de peneiras com aberturas de $9,50 \mathrm{~mm}, 4,75 \mathrm{~mm}$ e 2,36 mm. Além disso, foram fabricados briquetes com resíduos sem a realização de classificação granulométrica, ou seja, resíduos de variadas granulometrias misturados, cujo tratamento nomeou-se Sem Classificação (SC), como representado na Tabela 1.

Tabela 1. Classificação granulométrica dos resíduos de serragem de acordo coma malha das peneiras indicadas na coluna de tratamento e granulometria dos resíduos de serragem.

Tratamento Granulometria dos resíduos

\begin{tabular}{ll}
\hline $9,50 \mathrm{~mm}$ & $\geq 9,50 \mathrm{~mm}$ \\
$4,75 \mathrm{~mm}$ & $<9,5 \mathrm{~mm} \mathrm{e} \geq 4,75 \mathrm{~mm}$ \\
$2,36 \mathrm{~mm}$ & $<4,75 \mathrm{~mm} \mathrm{e} \geq 2,36 \mathrm{~mm}$ \\
$<2,36 \mathrm{~mm}$ & $<2,36 \mathrm{~mm}$ \\
$\mathrm{SC}$ & Sem classificação \\
Briquetes industriais & Sem classificação \\
\hline
\end{tabular}

Foram produzidos três briquetes laboratoriais por tratamento, cada um contendo $16 \mathrm{~g}$ de material, briquetados em condições controladas em uma briquetadeira do tipo Lippel modelo LB-32. A umidade do material estava entre $8 \%$ e $12 \%$, que é a faixa considerada ideal para produção de briquetes, com a máquina operando com temperatura de $120{ }^{\circ} \mathrm{C}$, a pressão utilizada foi de $1300 \mathrm{Bar}$, com tempo de prensagem de 5 minutos e de resfriamento de 6 minutos.

Briquetes industriais formaram outro tratamento, estabelecido como padrão comparativo para aqueles produzidos em laboratório. Esses briquetes são produzidos próximos à cerâmica para uso próprio e também são produzidos com resíduos de serraria. No entanto, os resíduos para produção desses não passam por classificação ou separação granulométrica e controle de umidade. Os briquetes industriais foram coletados na saída da matriz da briquetadeira em cinco ocasiões durante o turno de trabalho com intervalos de no mínimo 30 minutos.

As amostras de serragem e de briquetes industriais foram armazenadas em embalagens hermeticamente fechadas, embaladas, acondicionadas e enviadas para o LABEM/UFV.

Os briquetes industriais foram fragmentados em pedaços menores para a determinação da densidade aparente e umidade. Para a determinação da composição química, análise química imediata e poder calorífico superior, o material foi triturado com o uso de um moinho do tipo Wiley seguindo a norma TAPPI 257 om-52 (Tappi 1994), posteriormente foi classificado por peneiras de 40 mesh e 60 mesh, utilizando-se para análises o material retido na peneira de 60 mesh.

A umidade dos materiais foi determinada a partir do método gravimétrico por secagem em estufa. Para tanto, os discos seccionados de briquetes industriais foram pesados inicialmente e os mesmos foram acondicionados em estufa à aproximadamente $100{ }^{\circ} \mathrm{C}$, e após estabilização da massa, pesou-se novamente para obter o peso seco e a umidade foi determinada a partir da equação 01 :

$$
U=\left(\frac{P_{u}-P_{s}}{P_{s}}\right) * 100
$$

Em que: $\mathrm{U} \%=$ Teor de umidade (\%); $\mathrm{Pu}=$ Peso da amostra úmida $(\mathrm{g})$ e Ps = Peso da amostra seca $(\mathrm{g})$.

O poder calorífico superior dos resíduos e briquetes industriais foi determinado a partir de duplicatas, utilizando uma bomba calorimétrica modelo IKA C-200, seguindo a metodologia proposta pela norma brasileira - NBR 8633 (ABNT 1984).

A densidade a granel dos resíduos de serragem foi obtida conforme a NBR 6922 (ABNT 1981), que diz respeito à distribuição dos resíduos em um recipiente de madeira com dimensões conhecidas, as três amostras foram pesadas e em seguida determinou-se a densidade a granel a partir da relação entre a massa do material e o volume conhecido da caixa, utilizando-se a equação 02 :

$$
D G=\frac{m}{v}
$$

Em que: $\mathrm{DG}=$ Densidade a granel $\left(\mathrm{kg} \cdot \mathrm{m}^{-3}\right) ; \mathrm{m}=$ Massa $(\mathrm{kg})$ e $\mathrm{v}=$ Volume $\left(\mathrm{cm}^{3}\right)$.

A densidade energética dos resíduos e briquetes industriais foi determinada a partir dos valores conhecidos da densidade relativa aparente e do poder calorífico útil do mesmo, utilizando-se a equação 03 : 


$$
D E=D A x P C U
$$

Em que: $\mathrm{DE}=$ Densidade energética $\left(\mathrm{Gcal}^{-3} \mathrm{~m}^{-3}\right)$ DA $=$ Densidade aparente $\left(\mathrm{g} \cdot \mathrm{cm}^{-3}\right) ; \mathrm{PCU}=$ Poder calorífico útil $\left(\mathrm{kcal} \cdot \mathrm{kg}^{-3}\right)$.

A densidade aparente dos briquetes industriais foi determinada a partir da imersão em mercúrio com o uso de uma balança hidrostática para a determinação do volume deslocado pelo método proposto por Vital (1984), como também as amostras foram pesadas para a obtenção da massa. O cálculo para obtenção da densidade relativa aparente se deu a partir da equação 04:

$$
D A=\left(\frac{m}{v}\right) * D_{m e r}
$$

Em que: DA $=$ Densidade aparente $\left(\mathrm{g} \cdot \mathrm{cm}^{-3}\right) ; \mathrm{m}=\operatorname{Massa}(\mathrm{g}) ; \mathrm{v}=$ Volume $\left(\mathrm{cm}^{3}\right)$ e $\mathrm{D}_{\text {mer }}=$ Densidade do mercúrio $\left(\mathrm{g} \cdot \mathrm{cm}^{-3}\right)$.

A determinação do teor de extrativos foi realizada em duplicada seguindo a norma TAPPI 264 om-88, seguindo a equação 05:

$$
E x t=\left(\frac{P A S_{\text {ext }}}{P_{S}}\right) * 100
$$

Em que: Ext $=$ Teor de extrativos $(\%) ;$ PAS $_{\text {ext }}=$ Peso absolutamente seco de extrativos $(\mathrm{g}) ; \mathrm{P}_{\mathrm{s}}=$ Peso seco da amostra $(\mathrm{g})$.

A determinação da lignina se deu a partir do método Klason, modificado de acordo com o procedimento proposto por Gomide e Demuner (1986). Do filtrado restante da análise da lignina Klason foi realizada a leitura em espectrofotômetro, para determinação da lignina solúvel em ácido, conforme Goldschimid (1971).

A lignina total em porcentagem é obtida a partir do somatório dos teores de lignina solúvel e insolúvel, conforme expressa a equação 06 :

$$
L T=L_{s} L_{i}
$$

Em que: $\mathrm{LT}=$ Lignina Total; $\mathrm{L}_{\mathrm{s}}=$ Lignina solúvel; $\mathrm{L}_{\mathrm{i}}=$ Lignina insolúvel.

O teor de holocelulose foi obtido por diferença a partir dos valores determinados da quantidade de extrativos e lignina total, conforme a equação 07 :

$$
\text { Holo }=100-(L T+E x t)
$$

Em que: Holo $=$ Teor de holocelulose $(\%) ; \mathrm{LT}=$ Teor de lignina total $(\%)$; Ext $=$ Teor de extrativos $(\%)$.

A análise química imediata dos resíduos e dos briquetes industriais foi realizada seguindo a norma NBR 8112 (ABNT, 1986) para determinação do teor de materiais voláteis, cinzas e carbono fixo.

A resistência à compressão plana dos briquetes foi avaliada em uma máquina de ensaio mecânico universal, adaptando a NBR 7190 (ABNT, 1997), onde as amostras foram submetidas à força de compressão continuamente de $3,5 \mathrm{~mm}$. $\mathrm{min}^{-1}$ até que não oferecessem resistência, ocorrendo a ruptura. Os valores de resistência foram obtidos a partir de um software ligado a máquina que considera a área da amostra e a força da mesma oferecida até se romper.

As análises estatísticas dos dados foram realizadas utilizando o software STATISTICA 10 (STATSOFT, INC, 2012). Os dados foram submetidos aos testes de Lilliefors e Cochran para testar a normalidade e homogeneidade das variâncias, respectivamente. Em seguida, procedeu-se à análise de variância pelo teste $\mathrm{F}$, sendo as médias comparadas pelo teste de Tukey $(\mathrm{p} \leq 0,05)$.

\section{Resultados e discussão}

As propriedades físicas dos resíduos madeireiros variaram em função da posição de coleta na pilha de estocagem. Para densidade a granel houve aumento da mesma do topo para a base da pilha de estocagem (Tabela 2). Possivelmente, isso pode ser explicado pelo fato dos resíduos menores movimentarem na pilha de estocagem e se encaixarem mais facilmente entre os espaços vazios causados pelo acúmulo de resíduos maiores.

Quanto à umidade, pode-se observar que as três coletas apresentaram-se significativamente diferentes, sendo que a coleta na parte intermediária apresentou maior umidade. Esse fator pode ser explicado pela existência de menor superfície de contato dos resíduos nessa posição de coleta com o solo e o ar, como no caso das partes superior e inferior da pilha. Pinheiro et al. (2005) recomendam cuidados ao se armazenar os resíduos, devido à heterogeneidade dos mesmos e a baixa densidade a granel, o que pode influenciar negativamente suas características energéticas pelo ganho de umidade.

O poder calorífico superior foi maior para os resíduos coletados nas partes intermediária e inferior da pilha e apresentou relação direta com as características de densidade energética. Esta última seguiu o mesmo padrão observado anteriormente para o poder calorífico superior, onde apresentou-se maior na parte inferior da pilha, que não diferiu estatisticamente da parte intermediária, no entanto, apresentou diferenças estatísticas da parte superior (Tabela 2).

Tabela 2. Valores médios de densidade a granel (DG), umidade (U), poder calorifico superior (PCS) e densidade energética (DE) dos resíduos madeireiros em função do ponto de coleta na pilha de estocagem

\begin{tabular}{lcccc}
\hline \multirow{2}{*}{$\begin{array}{l}\text { Ponto de } \\
\text { coleta }\end{array}$} & \multicolumn{4}{c}{ Propriedades físicas } \\
\cline { 2 - 5 } & $\begin{array}{c}\mathrm{DG} \\
\left(\mathrm{kg} \cdot \mathrm{m}^{-3}\right)\end{array}$ & $\begin{array}{l}\mathrm{U} \\
(\%)\end{array}$ & $\begin{array}{c}\text { PCS } \\
\left(\mathrm{kcal}^{\mathrm{k}} \mathrm{kg}^{-1}\right)\end{array}$ & $\begin{array}{c}\mathrm{DE} \\
\left.\left(\mathrm{Gcal}^{-3}\right)^{-3}\right)\end{array}$ \\
\hline Superior & $173 \mathrm{~b}^{1 /}$ & $12,0 \mathrm{~b}$ & $4704 \mathrm{~b}$ & $0,81 \mathrm{~b}$ \\
Intermediária & $187 \mathrm{~b}$ & $19,0 \mathrm{a}$ & $4759 \mathrm{a}$ & $0,89 \mathrm{ab}$ \\
Inferior & $231 \mathrm{a}$ & $11,0 \mathrm{c}$ & $4783 \mathrm{a}$ & $1,10 \mathrm{a}$ \\
\hline${ }^{1 /}$ Médias seguidas das mesmas letras na coluna não diferem entre si $(\alpha=0,05)$ \\
pelo teste Tukey.
\end{tabular}

Os valores médios obtidos para as propriedades químicas dos resíduos madeireiros foram muito próximos daqueles observados para os briquetes industriais, indicando que essas propriedades não sofrem alterações significativas durante o processo de briquetagem (Tabela 3).

$\mathrm{O}$ teor de extrativos dos resíduos diferiu significativamente em função do ponto de coleta. Os resíduos da parte superior apresentaram maior teor de extrativos em sua composição, enquanto que os da parte intermediária apresentaram o menor teor.

Os teores de holocelulose diferiram de acordo com a posição de coleta, sendo que a parte intermediária apresentou o maior teor (Tabela 3). Os teores observados são coerentes com outros estudos realizados, como por exemplo, Rodrigues (2010) que observou teor de holocelulose em finos de madeira de $67,8 \%$ e Horst et al. (2015) que encontrou um teor de 62,9\% para serragem de madeira.

Os teores de lignina total, de cinzas e de carbono fixo não diferiram entre as três posições de coleta na pilha de estocagem. Essas propriedades apresentaram teores médios de $35,73 \%, 1,91 \%$ e $16,91 \%$, respectivamente (Tabela 3 ).

Tabela 3. Valores médios de extrativos (E), lignina total (LT), holocelulose (Holo) materiais voláteis (MV), cinzas (CZ) e carbono fixo $(\mathrm{CF})$ dos resíduos madeireiros em função do ponto de coleta na pilha de estocagem, em comparação com o briquete industrial (padrão). 


\begin{tabular}{lllllll}
\hline \multirow{2}{*}{$\begin{array}{l}\text { Ponto de } \\
\text { coleta }\end{array}$} & $\begin{array}{l}\mathrm{E} \\
(\%)\end{array}$ & $\begin{array}{l}\mathrm{LT} \\
(\%)\end{array}$ & $\begin{array}{l}\text { Holo } \\
(\%)\end{array}$ & $\begin{array}{l}\text { MV } \\
(\%)\end{array}$ & $\begin{array}{l}\mathrm{CZ} \\
(\%)\end{array}$ & $\begin{array}{l}\mathrm{CF} \\
(\%)\end{array}$ \\
\hline Superior & $5,8 \mathrm{~b}^{1 /}$ & $36,6 \mathrm{a}$ & $57,5 \mathrm{~b}$ & $79,17 \mathrm{~b}$ & $2,5 \mathrm{a}$ & $18,3 \mathrm{a}$ \\
Intermediária & $2,1 \mathrm{c}$ & $36,2 \mathrm{a}$ & $63,6 \mathrm{a}$ & $81,9 \mathrm{a}$ & $1,4 \mathrm{a}$ & $16,1 \mathrm{a}$ \\
Inferior & $6,6 \mathrm{a}$ & $34,3 \mathrm{a}$ & $57,2 \mathrm{~b}$ & $82,5 \mathrm{a}$ & $1,8 \mathrm{a}$ & $16,3 \mathrm{a}$ \\
\hline $\begin{array}{l}\text { Briquete } \\
\text { industrial }\end{array}$ & 7,2 & 40,1 & 52,7 & 78,11 & 5,6 & 16,3 \\
\hline $\begin{array}{l}1 / \text { Médias seguidas das mesmas letras na coluna não diferem entre si } \\
(\alpha=0,05) \text { pelo teste Tukey. }\end{array}$
\end{tabular}

O teor de lignina é importante para a briquetagem, já que a mesma é responsável pela aglutinação das partículas do resíduo durante a compactação a partir de sua plasticização. Esse componente pode influenciar também na estabilidade térmica do material, já que o mesmo é o composto da madeira que se degrada mais lentamente durante a queima.

Os teores de lignina observados, tanto nos briquetes industriais quanto nos resíduos que foram utilizados para fabricação dos briquetes laboratoriais, são maiores que aqueles observados por Trugilho et al. (2001). Esses autores, ao trabalharem com clones de Eucalyptus sp., principal espécie madeireira utilizada na produção de energia no país, encontraram teor de lignina variando de $27 \%$ a $32 \%$. Esta diferença pode estar relacionada ao tipo de material utilizado no presente estudo, que advém de diferentes espécies da Caatinga.

Para materiais voláteis, as partes intermediária e inferior da pilha apresentaram os maiores teores. Os menores teores desses materiais foram observados na parte superior da pilha, isso deve-se ao fato de que esta encontra-se mais exposta, além de exercer a função de proteger as demais camadas de perder material volátil.

É importante destacar que os teores de materiais voláteis e poder calorífico apresentam-se maiores para as mesmas partes da pilha de resíduo. Essas variáveis têm relação direta já que são os materiais voláteis são os principais responsáveis pelo fornecimento de energia durante a combustão, sendo que, no entanto, a queima destes materiais ocorre de forma rápida (Rodrigues 2010). A redução da área superficial dos resíduos através da densificação pode melhorar esta característica, produzindo uma queima mais lenta.

Os teores de cinzas observados são semelhantes aos observados para materiais lenhosos, como por exemplo, em Tavares et al. (2015), Horst et al. (2015), Cruz et al. (2016) e Fernandes et al. (2017). É importante que o teor de cinzas seja o mais baixo possível, pois altos teores nos briquetes podem ocasionar incrustações e corrosão em caldeiras, podendo diminuir a potencialidade da produção energética e emitir poluentes (Capote 2012). De acordo com Canto (2009) as cinzas interferem tanto na eficiência como na economia da combustão da biomassa, logo o manejo das mesmas é uma questão importante no uso da biomassa florestal para energia.

O teor carbono fixo foi semelhante aos valores encontrados por Santos et al. (2011) em espécies da Caatinga. Esse bioma tem sofrido grande devastação, em parte, devido à retirada de madeira para energia. Aqueles autores ressaltam que combustíveis com elevado teor de carbono fixo são considerados materiais de elevado potencial calorífico, uma vez que a maior quantidade de energia é normalmente oferecida nessas condições, com queima mais lenta, o que promove maior eficiência na combustão do material.
$\mathrm{O}$ resíduo madeireiro coletado apresentou heterogeneidade granulométrica. Partículas menores que 2,36 $\mathrm{mm}$ representaram a maior parte do material retido. Já as partículas que ficaram retidas na peneira de $9,50 \mathrm{~mm}$ de abertura apresentaram-se em menor quantidade (Figura 1). Para fins energéticos, o tamanho das partículas é importante para a velocidade de reação de queima do material, visto que, quanto menor o tamanho das partículas, maior a superfície específica de contato e mais rápida a transformação da biomassa em energia.

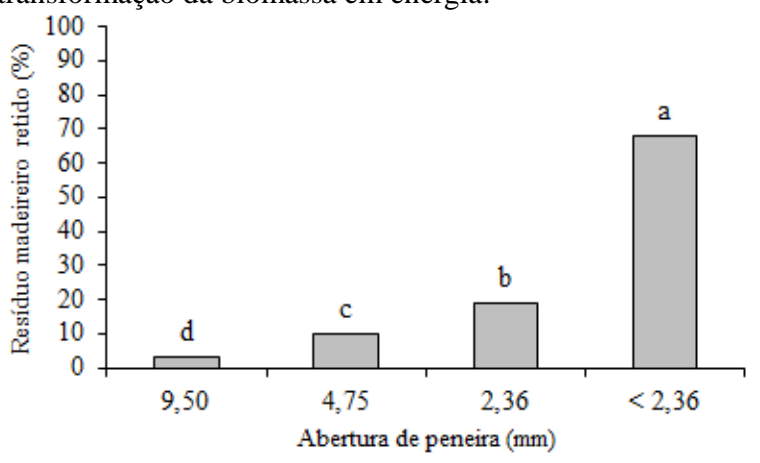

Figura 1. Porcentagem de resíduo madeireiro retido em peneiras com diferentes aberturas. Médias seguidas de mesmas letras não diferem entre si $(\alpha=0,05)$ pelo teste Tukey.

A densidade aparente dos briquetes laboratoriais foi semelhante àquela dos briquetes industriais (padrão). Entre os tratamentos dos briquetes laboratoriais, aqueles correspondentes ao tratamento 2,36 $\mathrm{mm}$ apresentaram a menor densidade aparente (Tabela 4).

Os briquetes do tratamento $2,36 \mathrm{~mm}$, juntamente com os do tratamento $<2,36 \mathrm{~mm}$, apresentaram maior teor de umidade, já os produzidos com resíduos de maior granulometria $(4,75$ e $9,5 \mathrm{~mm})$ e aqueles produzidos com resíduos sem classificação apresentaram menores teores. É importante destacar que os briquetes industriais apresentaram mais que duas vezes a umidade média observada nos briquetes laboratoriais (Tabela 4). Isso pode ser explicado pelo fato de antes de se compactar o resíduo na fábrica não ocorre controle do parâmetro, além disso, parte dos resíduos que chegam à fábrica é produzida pelo corte de madeiras com teores de umidade de médio a alto, acima do ponto de saturação das fibras.

Com relação à resistência à compressão plana dos briquetes, também não se observou um comportamento claro, já que a maior resistência à compressão foi observada para briquetes produzidos com resíduos de menor granulometria, para os de maior granulometria e para aqueles sem classificação. Esses dois últimos, no entanto não diferenciaram daqueles que foram produzidas com resíduos de granulometria intermediária (Tabela 4).

Tabela 4. Valores da densidade aparente (DA), umidade (U) e resistência à compressão plana (RCP) de briquetes laboratoriais, fabricados com resíduos madeireiros de diferentes granulometrias, em comparação com o briquete industrial (padrão).

\begin{tabular}{lccc}
\hline Tratamentos & DA $\left(\mathrm{kg} \cdot \mathrm{m}^{-3}\right)$ & $\mathrm{U}(\%)$ & $\begin{array}{c}\mathrm{RCP} \\
\left(\mathrm{kgf} . \mathrm{cm}^{-2}\right)\end{array}$ \\
\hline $\mathrm{SC}$ & $1,09 \mathrm{a}^{1 /}$ & $6,33 \mathrm{bc}$ & $154 \mathrm{ab}$ \\
$9,50 \mathrm{~mm}$ & $1,09 \mathrm{a}$ & $6,50 \mathrm{bc}$ & $153 \mathrm{ab}$ \\
$4,75 \mathrm{~mm}$ & $1,10 \mathrm{a}$ & $6,15 \mathrm{c}$ & $127 \mathrm{bc}$ \\
$2,36 \mathrm{~mm}$ & $1,01 \mathrm{~b}$ & $7,55 \mathrm{a}$ & $106 \mathrm{c}$ \\
$<2,36 \mathrm{~mm}$ & $1,08 \mathrm{a}$ & $7,22 \mathrm{a}$ & $188 \mathrm{a}$ \\
\hline Briquete & 1,02 & 17,75 & 198 \\
\hline industrial & & &
\end{tabular}


Em que: $\mathrm{SC}=$ Sem Classificação; ${ }^{1 /}$ Médias seguidas das mesmas letras na coluna não diferem entre si $(\alpha=0,05)$ pelo teste Tukey.

Os briquetes produzidos com resíduos de menor granulometria apresentaram resistência à compressão mais próxima àquela dos briquetes industriais. Deve-se levar em consideração que a pressão realizada pelas máquinas para compactar o material é diferente, visto que o equipamento utilizado em laboratório não consegue atingir a pressão de trabalho de uma máquina industrial.

Paula et al. (2011) realizando ensaios de resistência a compressão de briquetes, produzidos com resíduos semelhantes ao deste estudo, obtiveram uma variação de resistência mecânica de 167,74 a 224,29 kgf.cm-2. Já Furtado et al. (2010) ao estudarem a influência da matériaprima na qualidade de briquetes encontraram, para briquetes de serragem, valor médio de resistência à compressão plana de $115 \mathrm{kgf.cm}-2$, que é menor que o observado no presente estudo. Destaca-se que a força requerida no teste de resistência à compressão plana expressa a tensão máxima suportada pelo briquete até o seu rompimento e está relacionada com as forças de aderência entre as partículas do material que o constituem (Kaliyan e Morey 2009).

\section{Conclusões}

Os briquetes, tanto laboratoriais quanto industriais, produzidos com a utilização dos resíduos de serrarias, apresentam características como, poder calorífico superior, densidade aparente, resistência à compressão plana, teor de lignina e quantidade de carbono fixo, que os qualificam como importante fonte de energia para as indústrias de cerâmica, em razão dos valores considerados adequados para a biomassa energética.

A adoção de medidas de controle da matéria-prima, como granulometria de resíduos e umidade do material, para o processo de produção dos briquetes é fundamental para que os mesmos sejam eficientes em gerar energia calorífica.

\section{Referências}

Associação Brasileira de Normas Técnicas - ABNT (1997) NBR 7190: Projeto de estruturas de madeira. Rio de Janeiro.

Associação Brasileira de Normas Técnicas - ABNT (1986) NBR 8112: Carvão vegetal - análise imediata - método de ensaio. Rio de Janeiro.

Associação Brasileira de Normas Técnicas - ABNT (1984) NBR 8633: Carvão vegetal - Determinação do poder calorífico - Método de ensaio. Rio de Janeiro.

Associação Brasileira de Normas Técnicas - ABNT (1981) NBR 6922: Carvão vegetal: ensaios físicos de determinação da massa específica (densidade à granel). Rio de Janeiro.

Canto JL (2009) Colheita mecanizada de biomassa florestal para energia. Tese, Universidade Federal de Viçosa. 127p.

Capote FG (2012) Caracterização e classificação de coprodutos compactados da biomassa para fins energéticos. Dissertação, Universidade Federal do Paraná. 71p.

Costa TCC, Oliveira MAJ, Accioly LJO, Silva HBB (2009) Análise da degradação da Caatinga no núcleo de desertificação do Seridó (RN/PB). Revista Brasileira de Engenharia Agrícola e Ambiental, 13:961-974. doi: 10.1590/S1415-43662009000700020
Cruz BCC, Silva DA, Oliveira AS, Santos AB (2016)

Produção de briquetes de resíduos agrícolas e de serraria acrescido de resina de breu amarelo. Revista Brasileira de Energias Renováveis, 5(2):238-252. doi: 10.5380/rber.v5i2.44269

Fernandez BO, Gonçalves BF, Pereira ACC, Hansted ALS, Pádua FA, Da Róz AL, Yamaji FM (2017) Características mecânicas e energéticas de briquetes produzidos a partir de diferentes tipos de biomassa. Revista Virtual de Química, 9(1):29-38.

Furtado TS, Valin M, Brand MA, Bellote AFJ (2010) Variáveis do processo de briquetagem e qualidade dos briquetes de biomassa florestal. Pesquisa Florestal Brasileira, 30(62):101-106. doi: 10.4336/2010.pfb.30.62.101.

Goldschimid O. Ultraviolet spectra. In: Sarkanen KV, Ludwig CH. Lignins: occurrence, formation, structure and reactions. New York: John Wiley e Sons, 1971. p.241-298.

Gomide JL, Demuner BJ (1986) Determinação do teor de lignina na madeira: método Klason modificado. $O$ Papel, 47(1): 36-38.

Horst DJ, Ramírez JJ, Andrade Júnior PP, Serpe LF (2015) Assessing the lignin fraction extracted from Brazilian energy crops. American Journal of Environmental Sciences, 11(1):46-54. doi: 10.3844/ajessp.2015.46.54

Kaliyan K, Morey RV (2009) Factors affecting strength and durability of densified biomass products. Biomass \& Bioenergy, 33(3):337-359. doi: 10.1016/j.biombioe.2008.08.005

Pareyn FGC (2010) Os recursos florestais nativos e a sua gestão no Estado de Pernambuco: o papel do manejo florestal sustentável. In: Gariglio MA, Sampaio EVSB, Cestaro LA, Kageyama PY (ed) Uso sustentável e conservação dos recursos florestais da Caatinga. Brasília: SFB, p.99-115.

Paula LER, Trugilho PF, Rezende RN, Assis CO, Baliza AEB (2011) Produção de briquetes de resíduos lignocelulósicos. Pesquisa Florestal Brasileira, 31(66):103-112. doi: 10.4336/2011.pfb.31.66.103

Pinheiro GF, Rendeiro G, Pinho JT (2005) Densidade energética de resíduos vegetais. Biomassa \& Energia, 2(2):113-123.

Pontarolli AS, Los B, Waceliko C, Sviercowski DC, Oliveira EP (2013) Viabilidade da produção de briquetes a partir de resíduos agrícolas. Paraná Cooperativo Técnico Científico, 9(96):19-34.

Quirino WF (2007) Densificação de resíduos da biomassa. In: Workshop - Madeira Energética: Principais questões envolvidas na organização e no aperfeiçoamento do uso energético da lenha, Rio de Janeiro, Brasil.

Rodrigues VAJ (2010) Valorização energética de lodo biológico da indústria de polpa celulósica através da briquetagem. Dissertação, Departamento de Ciências Florestais, Universidade Federal de Viçosa. 117p.

Santos RC, Carneiro ACO, Castro RVO, Pimenta AS, Castro AFNM, Marinho IVM, Vilas Boas MA (2011) Potencial de briquetagem de resíduos florestais da região Seridó no Rio Grande do Norte. Pesquisa 
Florestal Brasileira, 31(68):285-294. doi:

10.4336/2011.pfb.31.68.285

Serviço Brasileiro de Apoio às Micro e Pequenas Empresas - SEBRAE. Diagnóstico da indústria de cerâmica vermelha do Rio Grande do Norte. Natal/RN, 2013.

Silva CA (2007) Estudo técnico-econômico da compactação de resíduos madeireiros para fins energéticos. Dissertação, Faculdade de Engenharia Mecânica, Universidade Estadual de Campinas. 68p.

Silva EF (2008) Diagnóstico do uso da lenha nas atividades agroindustriais do território do Seridó. Agência de Desenvolvimento Sustentável do Seridó-ADESE \& Cooperação Técnica Alemã-GTZ. Caicó/RN.

StatSoft, Inc. (2012) Statistica (data analysis software system), version 10.0.

Technical Association of The Pulp and Paper Industry TAPPI. Sampling and preparing wood for analysis, T257 om-52; Atlanta, 1994-1995.

Tavares MAME, Tavares SRL, Moreira IT (2015) A produção de briquetes para amenizar a pressão antrópica sobre o bioma Caatinga na região do BaixoAçu potiguar. Holos, 5:256-270. doi: 10.15628/holos.2015.2660

Trugilho PF, Lima JT, Mori AF, Lino AL (2001) Avaliação de clones de Eucalyptus para produção de carvão vegetal. Cerne, 7(2):104-114.

Vital BR (1984) Métodos de determinação da densidade da madeira. Viçosa - MG: SIF. 21p. 